\title{
Anomalous left ventricular chordae tendineae and pre-excitation Unusual cause of praecordial pansystolic murmur in a baby with fibroelastosis
}

\author{
M. Gueron and W. Cohen \\ From The Cardiac Laboratory, The Negev Central Hospital, Beer-Sheva, Israel
}

Clinical and necropsy findings are briefly described in a young child with pre-excitation who had a loud praecordial murmur. At necropsy, anomalous chordae tendineae were found in the left ventricular cavity stretching from the septum to the free wall in addition to severe endocardial fibroelastosis.

It is suggested that pre-excitation increased the intensity of the loud praecordial murmur caused by the stretched chordae.

The association of cardiac murmurs and preexcitation has been reported by several authors (Bergland et al., I966). On the other hand, transient praecordial murmurs have been associated with anomalous bands of the left ventricle (Roberts, 1969).

This report describes briefly a patient with extensive endocardial fibroelastosis in whom multiple anomalous left ventricular chordae and pre-excitation appeared to be the causes of a loud persistent praecordial murmur.

\section{Case report}

A 7-month-old baby boy was admitted to hospital for mild respiratory distress and diarrhoea of 3 days' duration. His previous history was not remarkable except for frequent respiratory 'difficulties' in the 3 months before admission. He was the product of a normal pregnancy and delivery.

The physical examination on admission revealed a well-nourished, well-developed patient in mild respiratory distress. There was no cyanosis or oedema. The femoral pulses were palpable and all peripheral pulses were normal. The blood pressure was $90 / 60 \mathrm{mmHg}$. The heart was enlarged; the cardiac impulse was not palpable. There were no thrills. The cardiac sounds were clear: the second sound over the second left intercostal space was single; $S_{1}$ and A2 were normal. A loud, rough, blowing grade $3 / 6$ pansystolic murmur, maximal at the left lower sternal border third to fifth intercostal spaces, was heard, radiat- ing toward the left axilla. The murmur remained unchanged with respiration. The liver was palpable two fingerbreadths below the right costal margin; the spleen was not enlarged.

The electrocardiogram, consistent with ventricular pre-excitation, showed a tachycardia of $200 / \mathrm{min}$ with a PR interval of $0.08 \mathrm{sec}$ and a QRS of $0.12 \mathrm{sec}$ (Fig. I).

The chest $x$-ray showed conspicuous cardiomegaly with mild lung congestion. The clinical findings were suggestive of a large ventricular septal defect in heart failure, though the electrocardiogram was consistent with fibroelastosis (Moss and Adams, I968).

The patient was given digitalis and was discharged with conspicuous improvement on the I5th hospital day; the physical findings and the electrocardiogram remained unchanged. Two months after discharge he was readmitted in severe heart failure and died on the seventh hospital day.

Necropsy revealed moderate cardiac enlargement: the heart weighed $90 \mathrm{~g}$. The left ventricular free wall thickness was $9 \mathrm{~mm}$ and the right $4 \mathrm{~mm}$. The endocardium was white, extremely thick with abundant fibroelastic tissue involving the entire left ventricle, the papillary muscles, and the left atrium. The left ventricular cavity was dilated. Three tendinal cords extended from the lower third of the left ventricular septal area to the left ventricular free wall with no connexion to the papillary muscles, the other chordae, or the mitral valve leaflets tissue (Fig. 2). Microscopical examination revealed extensive interstitial myocardial fibrosis extending from the area surround- 


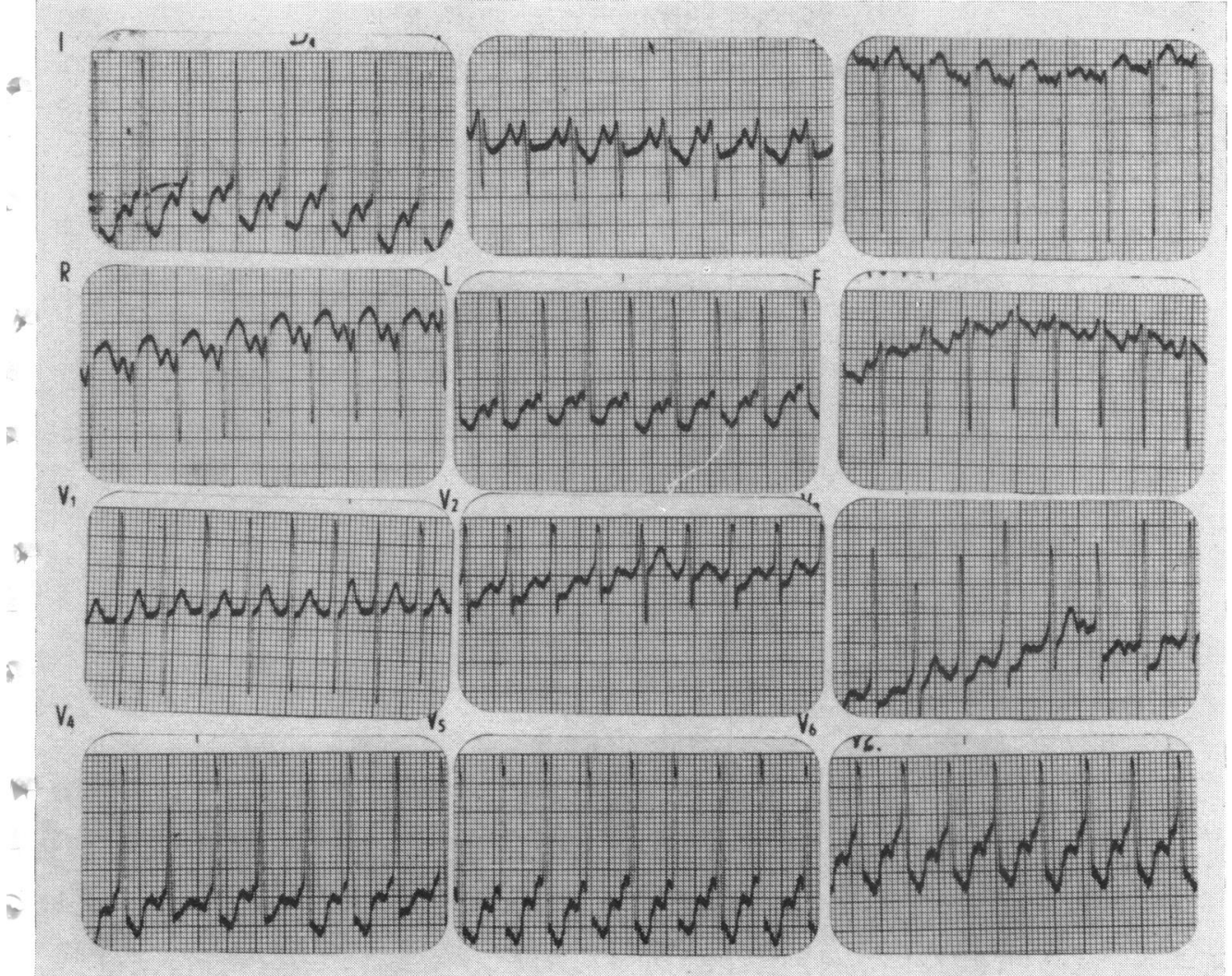

FIG. I Pre-excitation with a mean $Q R S$ axis in the frontal plane directed superiorly to the left. The ST sagging was consistent with digitalis effect.

ing the blood vessels. The endocardium was very thick with fibroelastic tissue - consistent with endocardial fibrosis.

\section{Discussion}

The mechanism of the pansystolic murmur observed in our patient was not clear; however, two factors were believed to be con-

- tributory: the anomalous bands and the left ventricular dilatation. Dilatation of the left ventricular cavity from any cause resulting in stretching of the atrioventricular ring and papillary muscles may be responsible for a praecordial murmur. On the other hand, it has been emphasized that anomalous left - ventricular bands may cause fleeting praecordial murmurs (Roberts, 1969). In our patient the murmur was not transient since even when the patient was fully compensated the ventricular dilatation persisted, confirming the mechanism advanced by Roberts (I969).

It has been suggested that pre-excitation may alter haemodynamics (Bergland et al.,
FIG. 2 Gross picture of the left ventricle with the abnormal chordae.

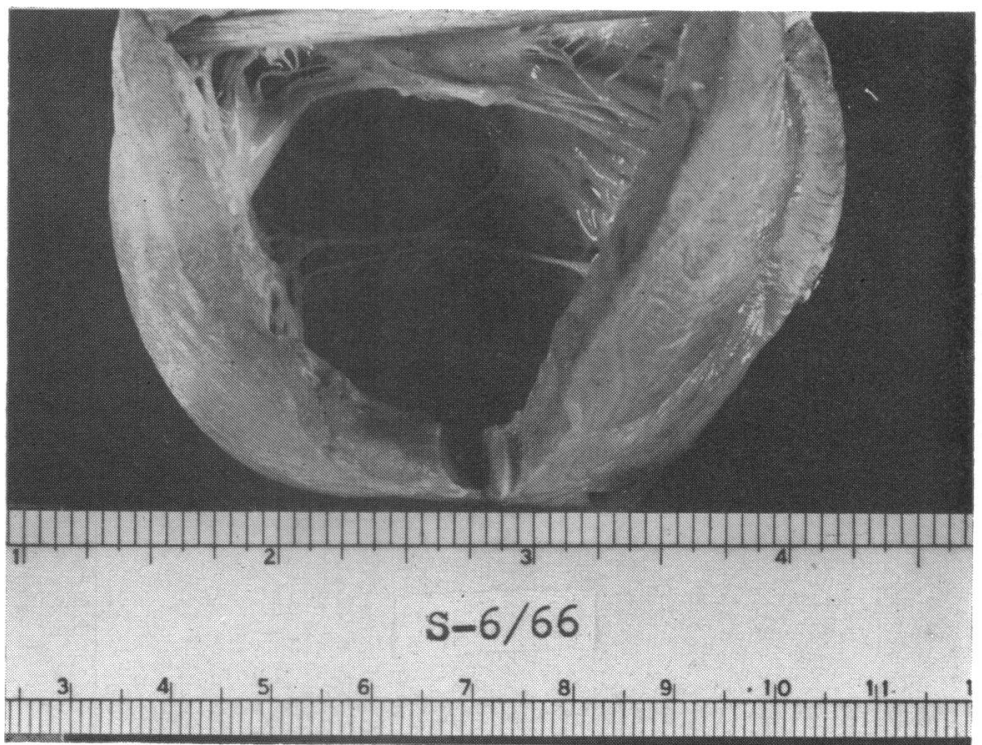


1966); however, the mechanism by which it increases the intensity of organic murmurs is not known. In our patient, it is quite possible that pre-excitation altered the intensity of the pansystolic murmur caused mainly by abnormal insertion of chordae tendineae in the left ventricular cavity.

\section{References}

Bergland, J. M., Rucker, W. R., Reeves, J. T., and Surawicz, B. (1966). Pre-excitation as a cause of appearance and increased intensity of systolic murmurs. Circulation, 33, 131.

Moss, A. J., and Adams, F. H. (1968). Heart Disease in Infants, Children and Adolescents. William and Wilkins, Baltimore.

Roberts, W. C. (1969). Anomalous left ventricular band. An unemphasized cause of a precordial musical murmur. American fournal of Cardiology, 23, 735 .

Requests for reprints to Dr. M. Gueron, Cardiopulmonary Laboratory, The Negev Central Hospital, P.O.B. I5r, Beer-Sheva, Israel. 